

\title{
Simulation of a macrosegregation benchmark in a cylindrical coordinate system with a meshless method
}

Vanja Hatič, Martín Cisternas Fernández, Boštjan Mavrič, Miha Založnik, Hervé Combeau, Božidar Šarler

\section{- To cite this version:}

Vanja Hatič, Martín Cisternas Fernández, Boštjan Mavrič, Miha Založnik, Hervé Combeau, et al.. Simulation of a macrosegregation benchmark in a cylindrical coordinate system with a meshless method. International Journal of Thermal Sciences, 2019, 142, pp.121-133. 10.1016/j.ijthermalsci.2019.04.009 . hal-02151405

\section{HAL Id: hal-02151405 \\ https://hal.univ-lorraine.fr/hal-02151405}

Submitted on 8 Jun 2019

HAL is a multi-disciplinary open access archive for the deposit and dissemination of scientific research documents, whether they are published or not. The documents may come from teaching and research institutions in France or abroad, or from public or private research centers.
L'archive ouverte pluridisciplinaire HAL, est destinée au dépôt et à la diffusion de documents scientifiques de niveau recherche, publiés ou non, émanant des établissements d'enseignement et de recherche français ou étrangers, des laboratoires publics ou privés. 


\title{
Simulation of a macrosegregation benchmark in a cylindrical coordinate system with a meshless method
}

\author{
Vanja Hatiča ${ }^{\mathrm{a}}$, Martín Cisternas Fernández ${ }^{\mathrm{b}}$, Boštjan Mavričc ${ }^{\mathrm{a}, \mathrm{c}}$, Miha \\ Založnik ${ }^{\mathrm{b}}$, Hervé Combeau ${ }^{\mathrm{b}}$, Božidar Šarler ${ }^{\mathrm{a}, \mathrm{c}}$ \\ ${ }^{a}$ Institute of Metals and Technology, Lepi pot 11, SI-1000 Ljubljana \\ ${ }^{b}$ Université de Lorraine, CNRS, IJL, F-54000 Nancy, France \\ ${ }^{c}$ Faculty of Mechanical Engineering, University of Ljubljana, Aškerčeva 6, SI-1000 \\ Ljubljana
}

\begin{abstract}
The main objective of the present paper is to define a new benchmark test for macrosegregation in axisymmetry and to verify a novel meshless method on it. The test case represents a solidification of $\mathrm{Al} 4.5 \mathrm{wt} \% \mathrm{Cu}$ alloy in two different types of geometries, a solid and a hollow cylinder, cooled at the vertical boundaries. The volume averaging method is used to formulate the coupled mass, energy, momentum, and species transport equations for solid-liquid flow. The lever rule is used for determination of liquid and solid fraction.

The meshless numerical approach, verified in this paper, is called the diffuse approximate method. The method is formed by using the weighted least squares approximation, where the second-order polynomial basis and Gaussians are used as trial and weight functions, respectively. The method is localised with the use of subdomains, each containing thirteen computational nodes. The explicit Euler scheme is used to perform the temporal integration. The fractional step method is used to couple the pressurevelocity fields. The stability of the method is attained by an adaptive shift of the computational node and Gaussian weight in the upstream direction.

Results are presented for three geometrically different simulations. The results are compared with the classical finite volume method. All results show a very good agreement with the finite volume method. The simulations
\end{abstract}

Email addresses: vanja.hatic@imt.si (Vanja Hatič), martin.cisternas-fernandez@univ-lorraine.fr (Martín Cisternas Fernández), bostjan.mavric@imt.si (Boštjan Mavrič), miha.zaloznik@univ-lorraine.fr (Miha Založnik), herve.combeau@univ-lorraine.fr (Hervé Combeau), bozidar.sarler@fs.uni-lj.si (Božidar Šarler) 
are performed on an uniform equidistant node arrangements of different node densities and the convergence of the node spacing is evaluated and compared. The results can also be used as a benchmark for other numerical methods.

Keywords: columnar solidification, macrosegregation benchmark, meshless diffuse approximate method, finite volume method, axisymmetry

\section{Introduction}

Macrosegregation is defined as an uneven distribution of the alloying elements on the macro-scale [1]. It is an undesired consequence of the solidification and represents one of the major casting defects. Modelling of the macrosegregation is therefore highly important for metallurgical and casting industries. Macrosegregation is caused by the transport of solute enriched (or depleted) liquid phase on the macroscopic scale. The root cause of the solute enrichment/depletion is different solubility in the liquid and in the solid phase. The main mechanisms for macroscopic transport of the rejected solute are: convection, shrinkage, floating of the solid grains, and forced flow. In general, each mechanism works in a different direction and with a different magnitude. The simulation of macrosegregation patterns is a highly complex, non-linear problem, and involves the solution of strongly coupled heat, mass, momentum and species transport equations. Furthermore, the problem exhibits multi-scale nature due to the coupling of the transport equations with the microsegregation model.

Meshless methods $[2,3]$ are in rapid development, especially in the last two decades, and are being applied to a growing number of various numerical applications. In contrast to the classical numerical approaches, such as finite volume or finite element method, the meshless methods do not require a computational mesh in form of a set of nodes, connected into a geometrical network. Only a set of uniformly or non-uniformly distributed computational nodes inside the global domain is used instead [2]. This greatly reduces the amount of effort required to generate the discretisation and thus allows a flexible application of the method to complex geometries. Furthermore, computation on scattered node arrangements, which are in addition adaptive in time and space [4], is also easier than in mesh-based numerical methods.

The numerical benchmark, presented and described in this paper, represents solidification of a binary alloy in a solid and hollow cylinder shaped cavity. The benchmark resembles the macrosegregation benchmark presented in [5]. A more complex macrosegregation benchmark, where the 
channel segregates are present, has also been solved and presented $[6,7,8]$. Yet, a related benchmark in axisymmetry, which requires a different implementation of the mathematical operators, has not been proposed and solved yet.

The test case represents an important step towards proper simulation of direct chill (DC) casting for round billets. Numerical models for simulation of DC casting and similar industrial processes in cylindrical coordinate systems have already been described in multiple papers $[9,10,11,12,13]$, but to the best of authors knowledge there is no benchmark test case combining solidification, melt-flow, and segregation in axisymmetry available in the literature.

Therefore the newly proposed benchmark is structured in a cylindrical coordinate system. The movement of the solid phase is not modelled, which is consistent with the previous benchmark cases simulated in Cartesian coordinate system $[5,6,7,8]$. The problem is solved with two completely different approaches; a) a meshless diffuse approximate method (DAM) with the explicit-Euler time-stepping and b) the finite volume method (FVM) with the implicit time-stepping. The FVM solution is obtained by using macroS3D which is a numerical solver developed using the open-source $\mathrm{C}++$ library OpenFOAM [14].

\section{Model formulation}

A volume-averaging formulation [9] is used to describe the two-phase solidification problem. The conservation equations are microscopically posed first, and along with the interphase boundary conditions form an exact description of the solidification process. The volume-averaging of the microscopic equations and the adjacent interphase conditions over a representative elementary volume results in a formulation that is free from the explicit shapes of the interphase boundaries and can thus be used for solving a spectrum of problems of practical interest. The governing macrosegregation equations are in the present work simplified to the largest possible degree in order to enable easier comparison of different numerical procedures. The liquid phase is modelled as an incompressible Newtonian fluid. Laminar flow is assumed. The solid and the liquid densities and the specific heats are constant and equal for both phases. The solid phase is static. The mushy zone is modelled with Darcy flow and Kozeny-Carman relation for permeability [15]. The buoyancy effect of the thermal and solutal density differences is modelled with the Boussinesq approximation. The dynamic viscosity of the liquid is assumed to be constant. The diffusion of the species on the 
macroscopic level is neglected for both phases. The local thermal equilibrium is assumed in the entire volume. The lever rule is used to calculate the liquid volume fraction. Each representative volume element is saturated. The listed assumptions result in the following volume-averaged governing equations for columnar solidification [16]

$$
\begin{gathered}
\nabla \cdot\left(g_{l} \mathbf{v}_{l}\right)=0, \\
\frac{\partial\left(g_{l} \mathbf{v}_{l}\right)}{\partial t}+\nabla \cdot\left(g_{l} \mathbf{v}_{l} \mathbf{v}_{l}\right)=-\frac{g_{l}}{\rho} \nabla p+\frac{\mu}{\rho} \nabla^{2}\left(g_{l} \mathbf{v}_{l}\right)-\frac{g_{l}^{2} \mu}{\rho K} \mathbf{v}_{l}+g_{l} \mathbf{b} ; \\
K=K_{0} \frac{g_{l}^{3}}{\left(1-g_{l}\right)^{2}} ; \\
\mathbf{b}=\mathbf{g}\left[1-\beta_{T}\left(T-T_{r e f}\right)-\beta_{C}\left(C_{l}-C_{r e f}\right)\right], \\
\rho \frac{\partial h}{\partial t}+\rho g_{l} \mathbf{v}_{l} \cdot \nabla h_{l}=\lambda \nabla^{2} T, \\
\frac{\partial C}{\partial t}+g_{l} \mathbf{v}_{l} \cdot \nabla C_{l}=0,
\end{gathered}
$$

where $t$ is the time, $\mathbf{v}_{l}$ is the liquid velocity, $\rho$ is the density, $g_{l}$ is liquid mass fraction, $p$ is the pressure, $\mu$ is the dynamic viscosity, $K_{0}$ is the Darcy constant, $\beta_{T}$ and $\beta_{C}$ are the temperature and the concentration volume expansion coefficients, respectively. $T$ is the temperature, $T_{r e f}$ is the reference temperature, $C_{l}$ is the liquid concentration, $C_{r e f}$ is the reference concentration, $\mathbf{g}$ is the gravitational acceleration vector, $h$ is the enthalpy, $h_{l}$ is the liquid enthalpy, $\lambda$ is the thermal conductivity, and $C$ is the average mixture concentration. The average concentration $C$ is obtained from the phase quantities as

$$
C=g_{l} C_{l}+\left(1-g_{l}\right) C_{s} .
$$

We assume that the solid and the liquid are locally in thermodynamic equilibrium. The phase concentration and temperature are thus related by

$$
\begin{aligned}
& C_{s}=k_{p} C_{l}, \\
& T=\left\{\begin{array}{cll}
\frac{h}{c_{p}} & \text { for } \quad h<c_{p} T_{s} \\
T_{f}+m_{l} C_{l} & \text { for } \quad c_{p} T_{s} \leq h<c_{p} T_{l}+L_{f} \\
\frac{h-L_{f}}{c_{p}} & \text { for } \quad c_{p} T_{l}+L_{f} \leq h
\end{array}\right.
\end{aligned}
$$


where $L_{f}$ is the latent heat of fusion, $k_{p}$ is the solid-liquid partition coefficient, $c_{p}$ is the specific heat capacity, $T_{f}$ is the fusion temperature of pure metal, and $m_{l}$ is the liquidus slope of a linearised binary phase diagram. $T_{l}$ and $T_{s}$ are the local liquidus and solidus temperatures, calculated from the phase diagram

$$
\begin{aligned}
& T_{s}=\max \left[T_{f}-m_{s} C, T_{e}\right], \\
& T_{l}=\max \left[T_{f}-m_{l} C, T_{e}\right] .
\end{aligned}
$$

The temperature, the liquid concentration and the liquid volume fraction are determined by using the constitutive relation for the volume-averaged enthalpy

$$
h=c_{p} T+g_{l} L_{f},
$$

and the lever rule. A quadratic equation for temperature is constructed, where only the following is physically valid

$$
\begin{aligned}
T & =\frac{-b-\sqrt{b^{2}-4 a c}}{2 a}, \\
a & =\left(1-k_{p}\right) c_{p}, \\
b & =\left(L_{f}-c_{p} T-h\right)\left(1-k_{p}\right)-L_{f}, \\
c & =\left(h T_{f}-L_{f} T_{f}\right)\left(1-k_{p}\right)+T_{l} L_{f} .
\end{aligned}
$$

The liquid fraction and liquid concentration are obtained by inserting the temperature in equation (8) and (6).

\section{Numerical benchmark definition}

The peculiar geometry is chosen in order to thoroughly test solidification model for the $r-z$ coordinate system, which is typically used for casting simulations of round geometries. Three cases of static casting are numerically evaluated in the present paper. All cases are of a cylindrical shape. Case 1 represents a solid cylinder, while Cases 2 and 3 are hollow cylinders with different inner and outer radii $r_{\text {in }}$ and $r_{\text {out }}$, respectively (see Figure 1). Axi-symmetry is assumed in all cases.

The results of the following three cases are presented in the present paper:

- Case 1: $r_{\text {in }}=0 \mathrm{~mm}, r_{\text {out }}=20 \mathrm{~mm}$, 
- Case 2: $r_{\text {in }}=10 \mathrm{~mm}, r_{\text {out }}=30 \mathrm{~mm}$,

- Case 3: $r_{\text {in }}=1000 \mathrm{~mm}, r_{\text {out }}=1020 \mathrm{~mm}$.

The height of all three cylinders is equal to $z_{\max }=20 \mathrm{~mm}$.

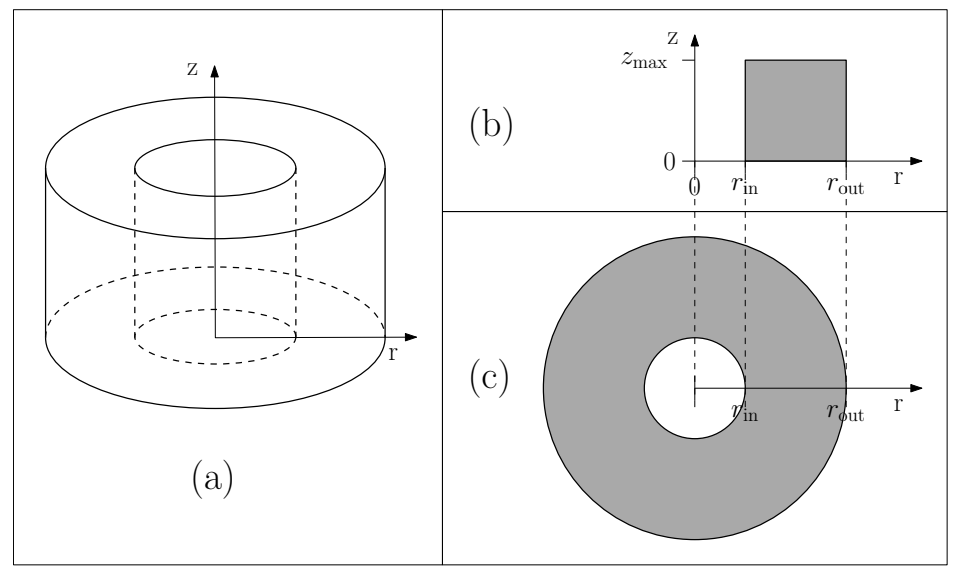

Figure 1: (a) 3D geometry of the hollow cylinder. (b) Cross section along the $r-z$ plane. (c) The top view.

The heat is extracted on vertical walls of the solid and hollow cylinders. The top and the bottom walls are thermally insulated. The solidifying metal is an Al4.5wt\% Cu alloy, with the material properties given in Table 1. 
Table 1: Material properties of Al4.5wt\% Cu alloy used in simulation.

\begin{tabular}{|l|l|l|r|}
\hline Property & Symbol & Unit & Value \\
\hline Specific heat capacity & $c_{p}$ & $\mathrm{~J} / \mathrm{kgK}$ & $1.00 \times 10^{3}$ \\
\hline Thermal conductivity & $\lambda$ & $\mathrm{W} / \mathrm{mK}$ & $1.92 \times 10^{2}$ \\
\hline Density & $\rho$ & $\mathrm{kg} / \mathrm{m}^{3}$ & $2.45 \times 10^{3}$ \\
\hline Dynamic viscosity & $\mu$ & $\mathrm{kg} / \mathrm{ms}$ & $1.2 \times 10^{-3}$ \\
\hline Gravity acceleration & $g$ & $\mathrm{~m} / \mathrm{s}^{2}$ & 9.80 \\
\hline Darcy constant & $K_{0}$ & $\mathrm{~m}^{2}$ & $5.56 \times 10^{-11}$ \\
\hline Latent heat & $L_{f}$ & $\mathrm{~J} / \mathrm{kg}$ & $4.00 \times 10^{5}$ \\
\hline Melting temperature of pure $\mathrm{Al}$ & $T_{f}$ & ${ }^{\circ} \mathrm{C}$ & 660 \\
\hline Eutectic temperature & $T_{e}$ & ${ }^{\circ} \mathrm{C}$ & 548 \\
\hline Eutectic concentration & $C_{e}$ & ${ }^{\circ} \mathrm{C}$ & 32.60 \\
\hline Liquidus slope & $m_{l}$ & ${ }^{\circ} \mathrm{C} / \mathrm{wt} \%$ & -3.43 \\
\hline Partition coefficient & $k_{p}$ & - & 0.173 \\
\hline Thermal expansion coefficient & $\beta_{T}$ & $1 / \mathrm{K}$ & $1.30 \times 10^{-4}$ \\
\hline Solutal expansion coefficient & $\beta_{C}$ & $1 / \mathrm{wt} \%$ & $-7.30 \times 10^{-3}$ \\
\hline Reference temperature & $T_{\mathrm{ref}}$ & ${ }^{\circ} \mathrm{C}$ & 465 \\
\hline Reference concentration & $C_{\mathrm{ref}}$ & $\mathrm{wt} \%$ & 4.50 \\
\hline
\end{tabular}

The initial temperature and concentration are constant and set to $T_{0}=$ $700{ }^{\circ} \mathrm{C}$ and $C_{0}=4.5 \mathrm{wt} \%$, respectively. The liquidus temperature is $644.565^{\circ} \mathrm{C}$. The liquid metal is at rest at the beginning of the simulation. The total duration of the simulation is $50 \mathrm{~s}$. Symmetry of the geometry and the fields coincide with the $z$-axis, therefore only the right half of the ingot is modelled. 


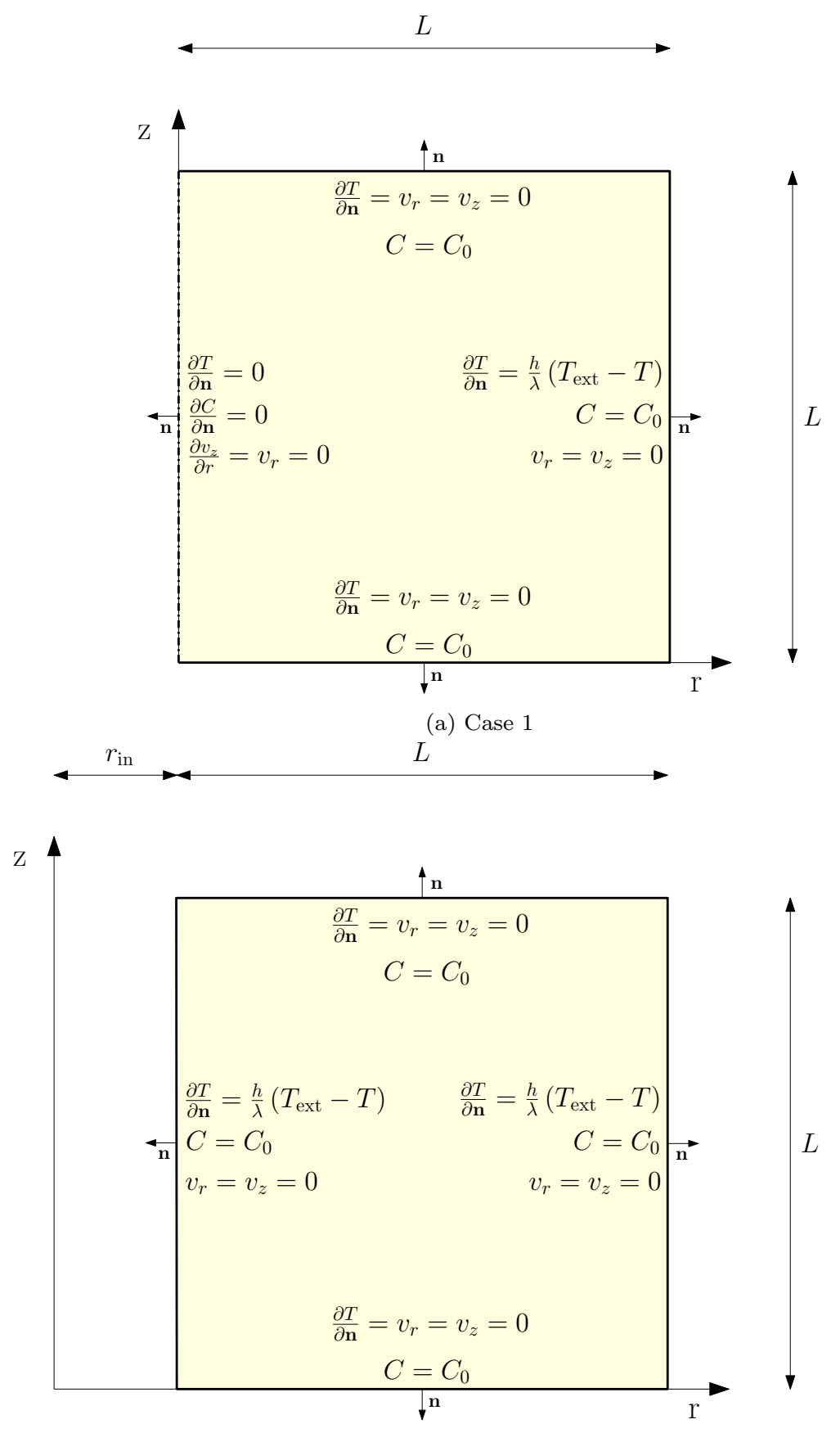

(b) Case 2 and 3

Figure 2: Boundary conditions for momentum, heat, and species transfer. 
Dirichlet type boundary condition with concentration $C_{0}$ is used everywhere except at the symmetry axis. This boundary condition can be imposed, as it is the solution of the species transport equation at the boundary nodes that correspond to walls. The no-slip velocity boundary condition is imposed everywhere except at the symmetry axis. The north and the south boundaries are thermally insulated and the temperature gradient in the normal direction is equal to zero at these boundaries. The east and the west boundaries are cooled and modelled with the Robin-type boundary condition, where the heat transfer coefficient equals to $500 \mathrm{~W} \mathrm{~K}^{-1} \mathrm{~m}^{-2}$ and the external temperature is set to $20{ }^{\circ} \mathrm{C}$. In Case 1 the west boundary is a symmetry axis and symmetry boundary conditions are used for all quantities instead. The cylinder geometry and the boundary conditions are displayed in Figure 2. Figure 3 shows the positions of the points and cross sections, where the transient and the final results are compared.

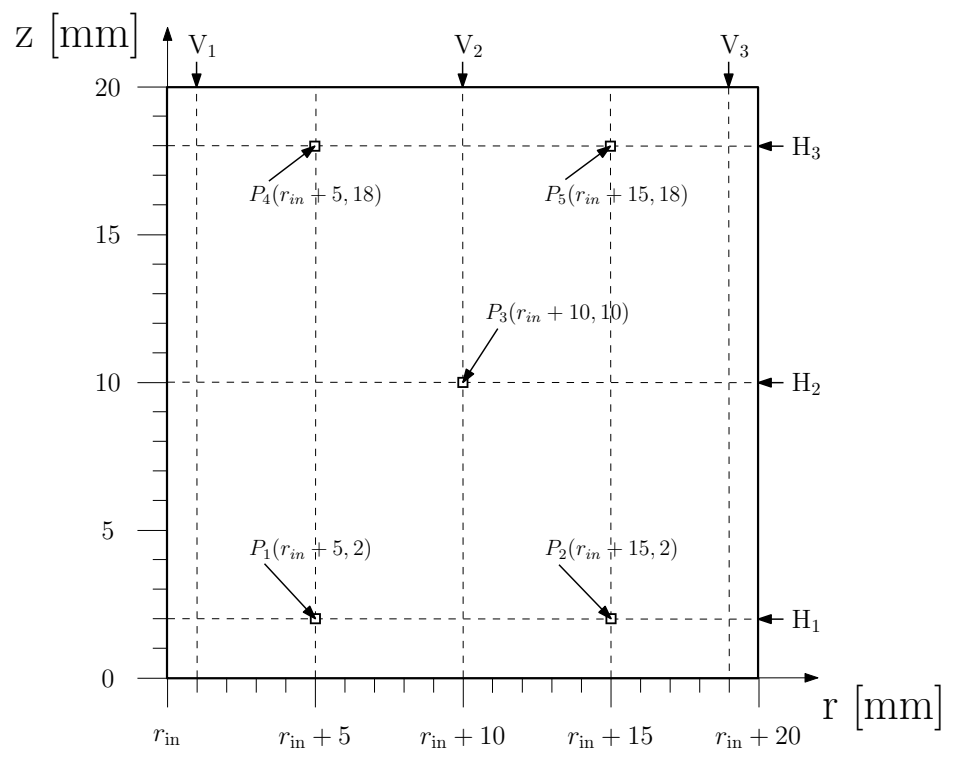

Figure 3: Geometry of the solidifying system. Three vertical and horizontal cross sections used for numerical verification are denoted with dashed line. Five sample points, used to show the time-evolution of solidification, are marked with the hollow squares.

\section{The numerical procedure}

The meshless diffuse approximate method is used for the spatial discretisation. DAM represents a strong form meshless method where the fields are represented by weighted least squares method on overlapping subdomains. 
The method has been first proposed in [17] and further developed mainly by the group of Professor H. Sadat $[18,19,20]$. The method shows a secondorder rate of convergence for the quadratic polynomial base [21].

The diffuse approximate method has been used by different research groups to solve a large spectrum of physical problems, such as convectivediffusive heat transfer problems [22], 3D fluid flow [23] and 2D fluid flow on an irregular domain [24], computation of hydromagnetic stagnation point flow in 2D [25], simulation of phase transformation with front tracking [26], and the phase field model simulation of Rayleigh-Taylor instability [27] and microjet flow in axisymmetry [28]. The numerical method has already been used to simulate the macrosegregation benchmark in a $x-y$ coordinate system [29]. Of all the listed problems solved with DAM, only the microjet flow has been simulated in the cylindrical coordinate system. An application to an industrial problem of low-frequency of electromagnetic DC casting [4] has also been performed with DAM in axisymmetry, however it is hard to verify the method in such complex situations.

Computational nodes are positioned inside and on the boundary of the domain. A local subdomain of its closest neighbouring nodes is assigned to each computational node. Figure 4 shows two examples of global domains. The first one (a) is of an arbitrary shape, which has non-uniform distribution of computational nodes. The second one (b) is a square cavity with uniform and equidistant distribution of nodes, which is used for computations in the present paper. The local subdomains for boundary and domain nodes are denoted with dotted and dashed line, respectively.

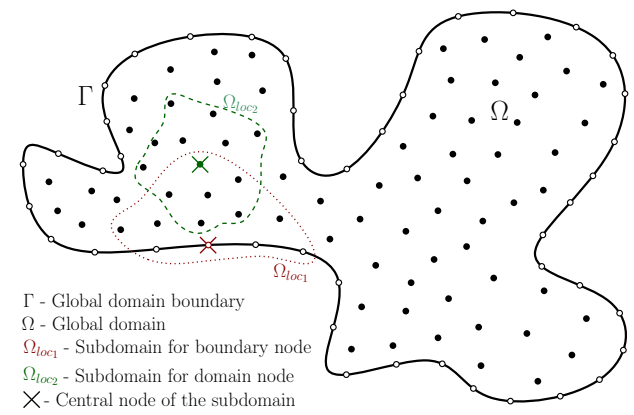

(a)

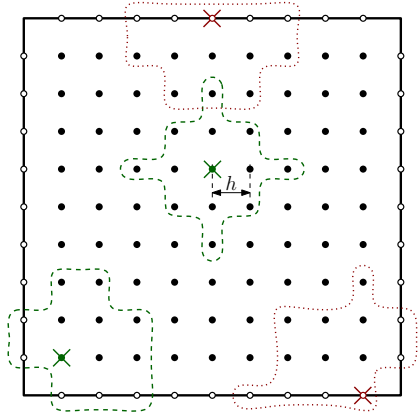

(b)

Figure 4: Scheme of the subdomains for domain and boundary computational nodes with thirteen local nodes for an arbitrarily shaped domain with non-uniform distribution of the nodes (a) and a rectangular geometry with uniform computational node arrangement (b).

The weighted least squares method is used to determine locally smooth 
and differentiable approximation of the discrete data on the overlapping subdomains. The approximation $\hat{f}^{l}$ of the given value $f$ in the chosen subdomain $l$ is given by the following expression

$$
\hat{f}^{l}(\mathbf{r})=\mathbf{p}\left(\mathbf{r}, \mathbf{r}_{l}\right) \cdot \boldsymbol{\alpha}^{l}=\sum_{j=1}^{J} p_{j}\left(\mathbf{r}, \mathbf{r}_{l}\right) \alpha_{j}^{l},
$$

where $\mathbf{r}_{l}$ is the position vector of the central node of the subdomain l. $\mathbf{p}\left(\mathbf{r}, \mathbf{r}_{l}\right)$ is the polynomial basis vector of size $J$ and $\boldsymbol{\alpha}^{l}$ is the vector of coefficients. The second-order basis is used in simulations (i.e. $J=6$ )

$$
\begin{aligned}
\mathbf{p}\left(\mathbf{r}_{i}, \mathbf{r}_{l}\right)= & {\left[1,\left(x_{1 i}-x_{1 l}\right),\left(x_{2 i}-x_{2 l}\right),\right.} \\
& \left.\left(x_{1 i}-x_{1 l}\right)\left(x_{2 i}-x_{2 l}\right),\left(x_{1 i}-x_{1 l}\right)^{2},\left(x_{2 i}-x_{2 l}\right)^{2}\right] .
\end{aligned}
$$

The approximation (10) is obtained with the weighted least squares approach, by minimization of the expression

$$
E^{l}=\sum_{i=1}^{n} \theta\left(\mathbf{r}_{i}, \mathbf{r}_{l}\right)\left[\hat{f}^{l}\left(\mathbf{r}_{i}\right)-f\left(\mathbf{r}_{i}\right)\right]^{2},
$$

where $n$ is the number of the nodes in the subdomain and $\theta$ is the Gaussian weight function. The weight function has a peak value of 1 at the chosen position $\mathbf{r}_{l}$ and decreases with increasing Euclidean distance from the node

$$
\theta\left(\mathbf{r}_{i}, \mathbf{r}_{l}\right)=\exp \left(-c \frac{\left\|\mathbf{r}_{i}-\mathbf{r}_{l}\right\|^{2}}{h^{2}}\right)
$$

with $h$ denoting the distance between the central node of the subdomain and its nearest neighbour. $h$ is used to scale the Euclidean distance by the local domain size. The free parameter of the weight function is $c$, which determines how gradual the decay of the Gaussian from 1 to 0 is. An upwind approach is required in order to stabilize the convective terms. This is particularly important in Equation (4), since the species transport exhibits pure advection. A detailed description of the upwind approach using the weight function is described in [4].

The derivative of the basis vector (11) has to be used in the approximation expression (10) and minimisation expression (12) at the nodes where 
the Neumann boundary conditions are implemented. The vector of coefficients for each subdomain $\boldsymbol{\alpha}^{l}$ is obtained from the solution of the system of equations

$$
\sqrt{\theta\left(\mathbf{r}_{i}, \mathbf{r}_{l}\right)} \mathbf{p}\left(\mathbf{r}_{i}, \mathbf{r}_{l}\right) \boldsymbol{\alpha}^{l}=\sqrt{\theta\left(\mathbf{r}_{i}, \mathbf{r}_{l}\right)} f\left(\mathbf{r}_{i}\right) ; \text { for every } i .
$$

The number of the nodes in the local neighbourhood has to at least match the size of the basis vector, which is equal to 6 for the case of quadratic polynomial base, in order to guarantee the solvability of Equation (14). The local neighbourhoods used in simulations contain thirteen nodes. The same number of the local nodes was used in our previous simulations with DAM $[4,29]$ for solidification problems.

The explicit-Euler time-stepping scheme is used for the spatial discretisation. The simple first order stepping performs well when it is used to solve thermo-fluid problems. This has already been confirmed by numerous benchmark cases [5, 8, 24, 29, 30, 31, 32, 33, 34].

\section{Numerical implementation}

The meshless model, described in the previous section, is written in Fortran 2008 and compiled into a 64-bit executable with Intel Visual Fortran compiler (XE 14).

The FVM reference results are obtained with macroS3D, a numerical solver developed using the OpenFOAM [14] platform, which was compiled using the GNU Compiler Collection (GCC). It uses an iterative method to couple the temperature, liquid fraction, solute concentration, liquid velocity and pressure. A two-iteration PISO algorithm is used in the velocitypressure coupling. The van Leer scheme is used for stabilisation of the advective terms. With respect to the linear solvers, a preconditioned conjugate gradient method is used for the pressure, while a Gauss-Seidel method is used for the temperature.

Operators used in conservation equations (1-4) have a different implementation for Cartesian and Cylindrical coordinate system. Definitions for gradient, divergence and Laplace operators in cylindrical coordinates are given in Appendix A.

\section{Time and spatial discretisation}

Simulations are performed on uniform distributions with the total number of nodes (DAM) and cells (FVM) equal to 40x40, 100x100, 140x140, 
$200 \times 200$, and $240 \times 240$. The time step is equal to $2 \times 10^{-5}$ s for DAM and $5 \times 10^{-4}$ s for FVM. The comparison of computational node arrangements for DAM and cell centres for FVM used in each of the numerical codes is shown in Figure 5. The comparison is shown for arrangement with the largest node spacing.



(a) DAM nodes

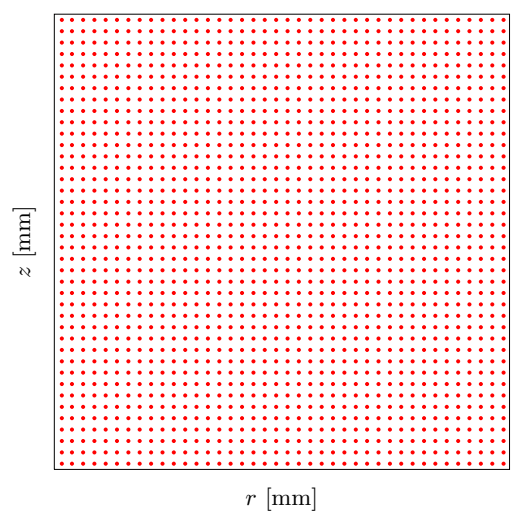

(b) FVM cell centres

Figure 5: Comparison of 40x40 discretisation used in DAM and FVM.

\section{Results}

The calculated transient of the solidification process is shown in Figures 6 to 8 . The time evolution of the fields is shown for five characteristic points. The positions of the points are given in Figure 3. DAM and FVM predictions are shown with the solid lines and discrete points, respectively. The temporal evolution of the liquid fraction, temperature and velocity is nearly the same for all characteristic locations in all three simulated geometries. From the concentration evolution it is observed that the pattern is the same for both numerical methods. There is a slight discrepancy in the magnitude of segregation in the observed points. The conservation of concentration field is evaluated due to this fact. It is estimated from the relative loss of the concentration $\left(\Delta V_{C}\right)$ in the entire volume of the geometry

$$
\Delta V_{C}=\frac{1}{V} \int \frac{\left(C-C_{0}\right)}{C_{0}} d V \approx \frac{\sum_{i=1}^{n} r \frac{\left(C_{i}-C_{0}\right)}{C_{0}} \Delta A_{i}}{\sum_{i=1}^{n} r \Delta A_{i}} .
$$

The values of $\Delta V_{C}$ are similar for Cases 1,2 and 3 and are equal to $4.108 \times 10^{-4}, 2.822 \times 10^{-4}$ and $2.466 \times 10^{-4}$ for DAM, respectively. All 
of the listed values are very small and it is confirmed that the species mass is globally conserved. The relative loss of the concentration in FVM simulations is even smaller, because it is a conservative method by construction, both locally and globally. The $\Delta V_{C}$ is equal to $1.629 \times 10^{-5}$, $2.511 \times 10^{-7}$ and $4.410 \times 10^{-6}$ for Cases 1,2 and 3 , respectively. 




(a) Liquid fraction

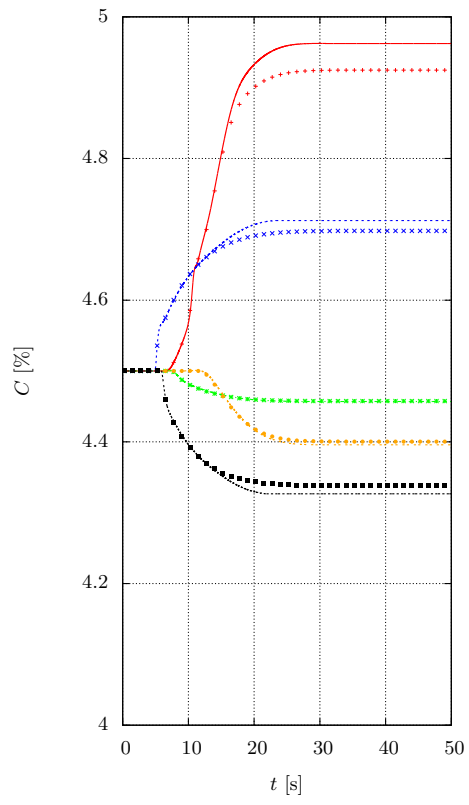

(c) Concentration

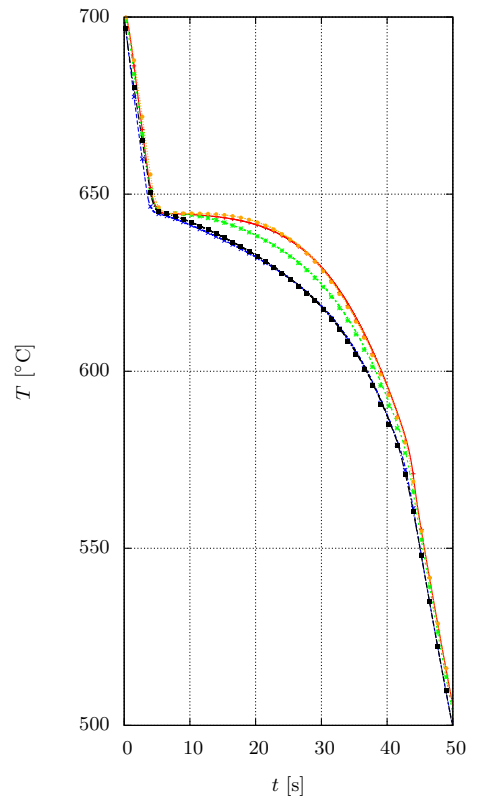

(b) Temperature

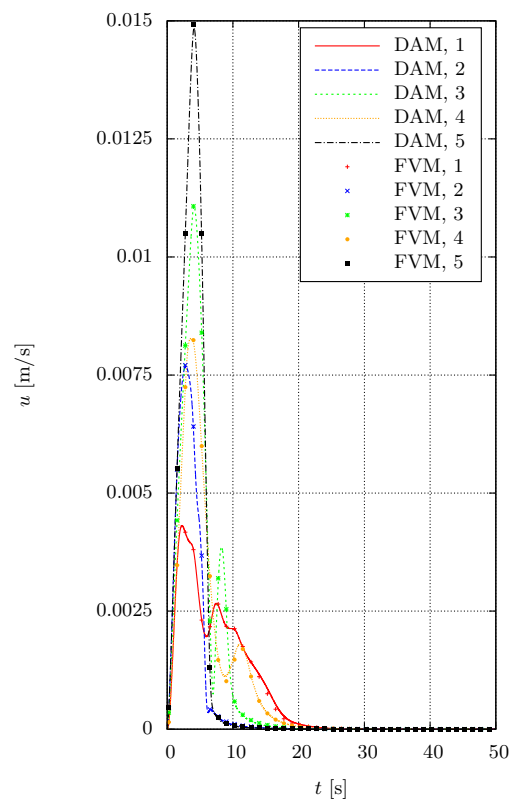

(d) Velocity magnitude

Figure 6: Time evolution of the results in points 1-5 for Case 1. Labels in (a), (b), and (c) are the same as in (d). Results are obtained on 240x240 computational node arrangement for both numerical methods. 


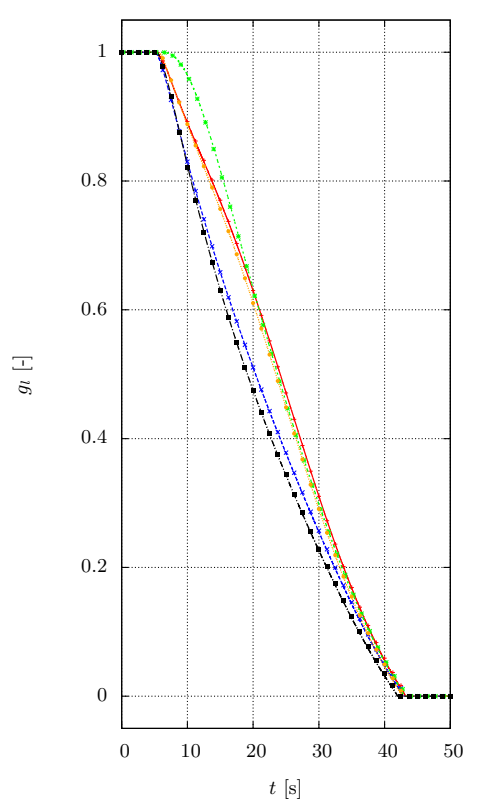

(a) Liquid fraction

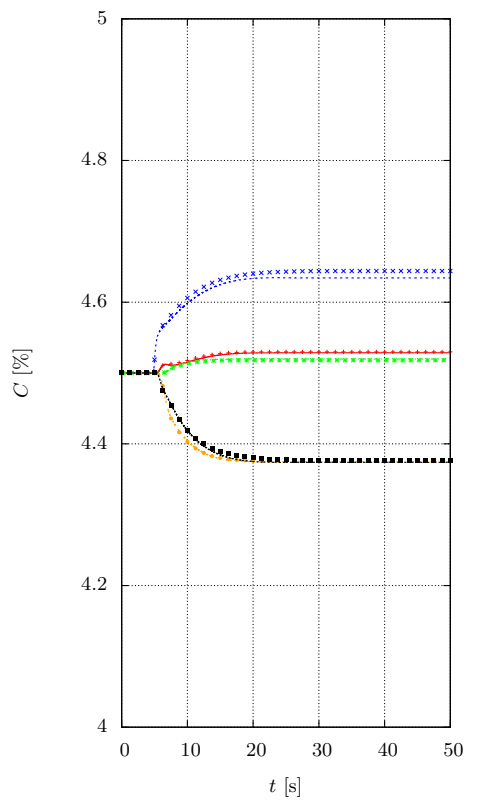

(c) Concentration

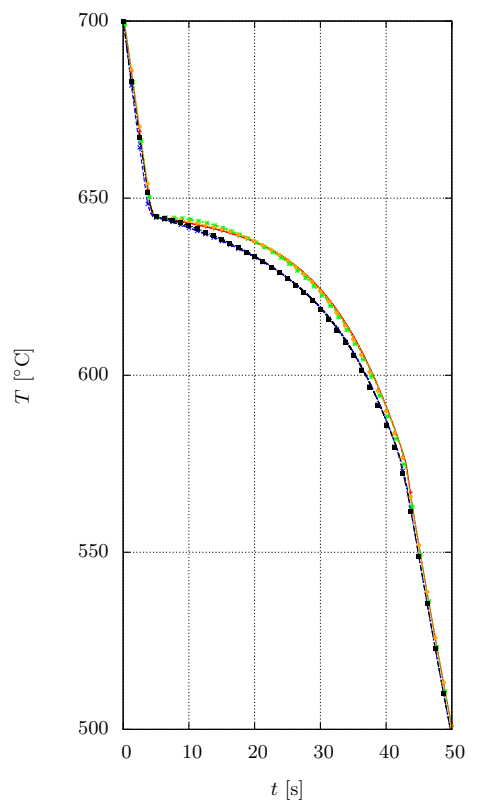

(b) Temperature

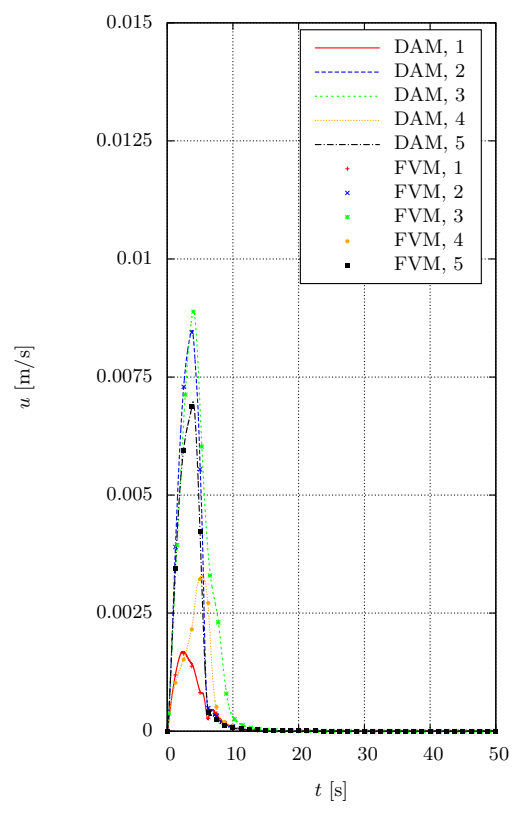

(d) Velocity magnitude

Figure 7: Time evolution of the results in points 1-5 for Case 2. Labels in (a), (b), and

(c) are the same as in (d). Results are obtained on 240x240 computational node arrangement for both numerical methods. 


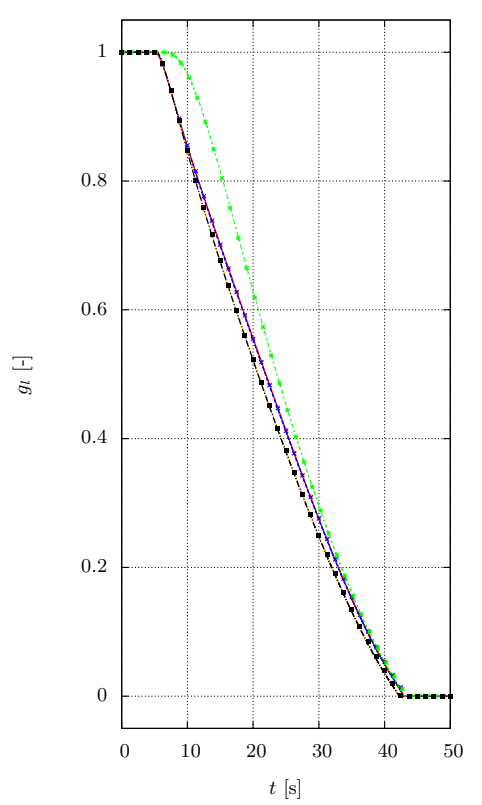

(a) Liquid fraction

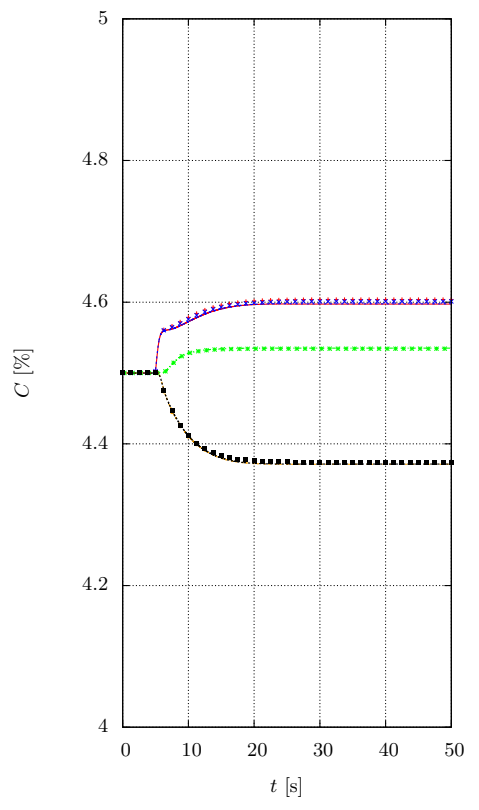

(c) Concentration



(b) Temperature

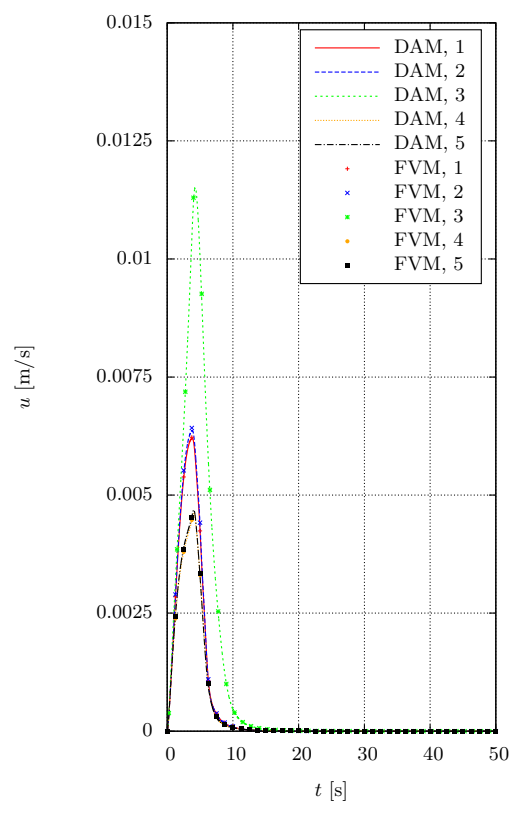

(d) Velocity magnitude

Figure 8: Time evolution of the results in points 1-5 for Case 3. Labels in (a), (b), and (c) are the same as in (d). Results are obtained on 240x240 computational node arrangement for both numerical methods. 
Solidification process is similar for all three cases. The melt flow is the most intensive after about $4 \mathrm{~s}$ of simulation time just before the solidification begins. The velocity magnitude at that point is about $1.0-1.5 \mathrm{~cm} / \mathrm{s}$ and is the largest for Case 1. The solidification starts near the vertical walls, at the area where the temperature first drops below liquidus temperature (644.5 ${ }^{\circ} \mathrm{C}$ ). The macrosegregation profile starts to form simultaneously. The solidification is finished after $42 \mathrm{~s}$. The macrosegregation evolution completes when the solid fraction is high enough to sufficiently restrict the melt flow in the mushy zone. This happens before the end of solidification, after about $25 \mathrm{~s}$, when the liquid fraction is equal to $\sim 0.4$ (see Figures 6 to 8 ). The macrosegregation pattern at that time is positive at the south and negative at the north part of the cavity, respectively.

The final macrosegregation patterns are presented with the contour plots in Figures 9 - 11. Blue and red colour are reserved for positive and negative macrosegregation, respectively. The colour-filled contour plots are shown only for DAM simulations, as the differences with FVM results are not visible to the naked eye. The limit between the positive and negative segregation values is denoted with the solid black isoline for DAM and with the dashed black isoline for FVM.

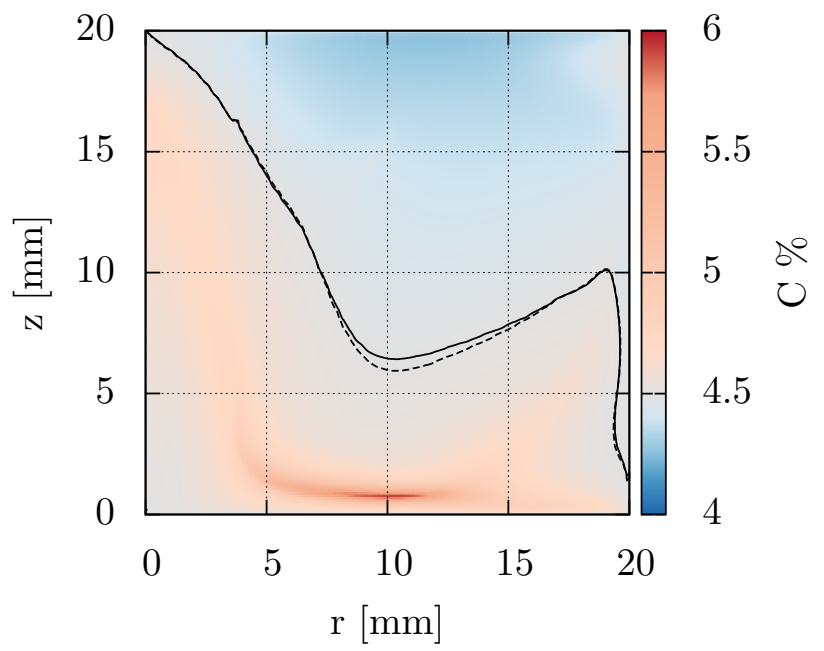

Figure 9: Final macrosegregation pattern for Case 1. 


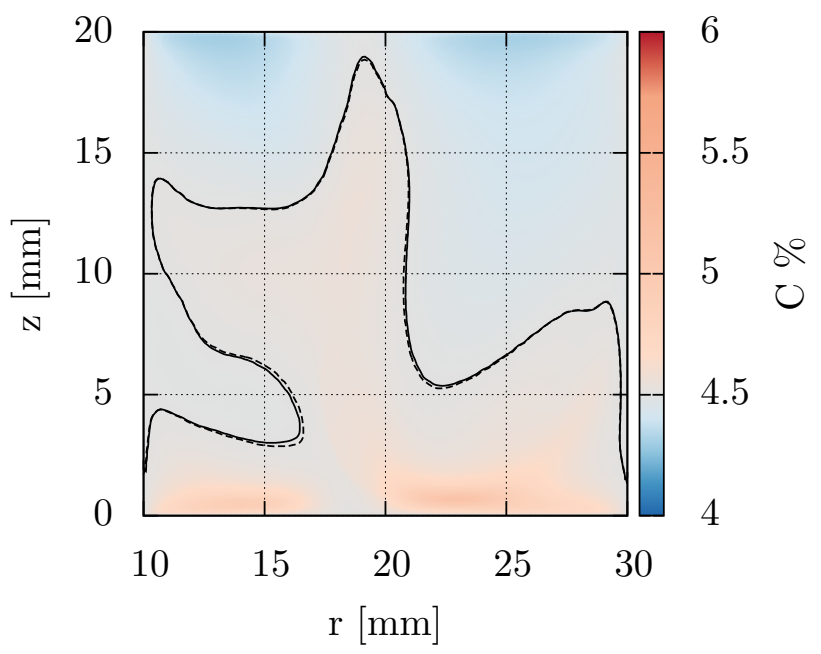

Figure 10: Final macrosegregation pattern for Case 2. 


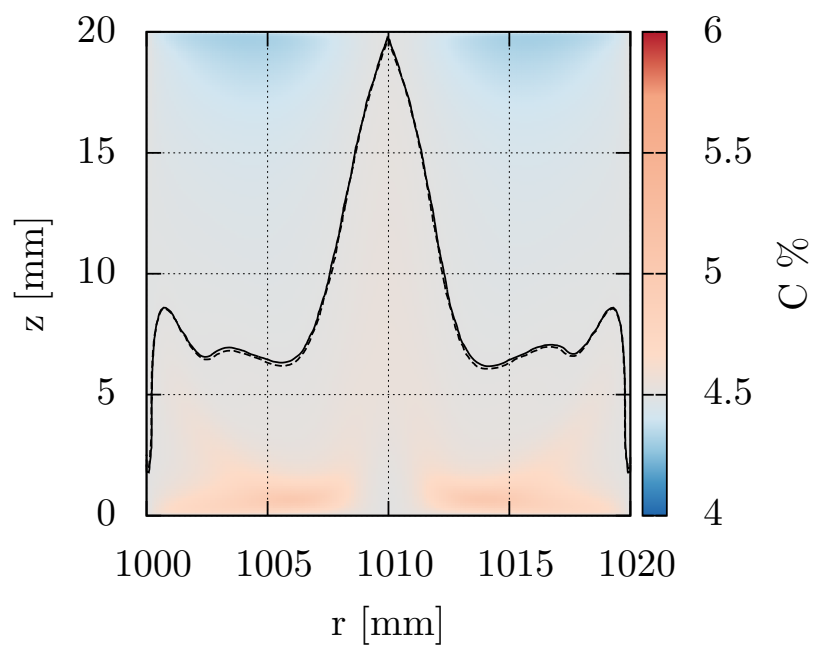

Figure 11: Final macrosegregation pattern for Case 3. 
Three different simulation Cases $(1,2,3)$ are distinguished by the value of $r_{\text {in }}$ and $r_{\text {out }}$. For the Case 1 and 2 the solidification is not symmetrical across the vertical line at the midpoint $\left(r_{\text {in }}+r_{\text {out }}\right) / 2$. This happens due to hollow cylinder geometry, which has a larger cooling surface on the outer wall. Consequently, the heat extraction is faster at the outer boundary. Yet, for Case 3, the difference between the inner and the outer radius is very small. The cooling rate is almost equal at both vertical walls. Therefore the solidification is practically symmetrical across the midpoint vertical line (at $r=1010 \mathrm{~mm})$. The results for Case 3 resemble the ones obtained for the macrosegregation in a square cavity [5].



Figure 12: Liquid fraction, temperature contours and velocity vectors of the melt flow for Case 1 at $t=10 \mathrm{~s}$. 


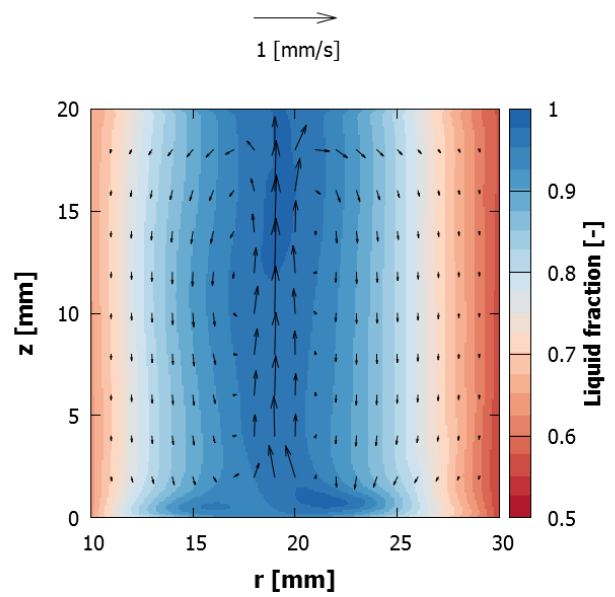

Figure 13: Liquid fraction, temperature contours and velocity vectors of the melt flow for Case 2 at $t=10 \mathrm{~s}$.

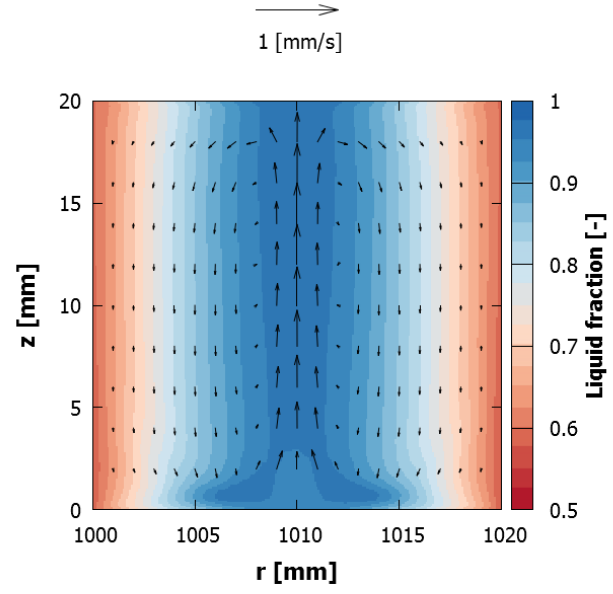

Figure 14: Liquid fraction, temperature contours and velocity vectors of the melt flow for Case 3 at $t=10 \mathrm{~s}$.

With the onset of solidification, the growing solid rejects solute into the liquid, reinforcing the thermal effect in the buoyancy driven flow, which is depicted in Figures 12-14. In Case 1, a clockwise circulation can be observed due to its symmetry condition on its west boundary, while in the other cases, there are two circulations in opposite sense of rotation. The formation of solute enriched channels, due to the liquid flow in the mushy zone, can be 
noticed close to the bottom wall and it follows the relation $\frac{\partial C}{\partial t} \sim \mathbf{v}_{l_{r}} \cdot \nabla T$, where $\mathbf{v}_{l r}$ is the horizontal component of the liquid velocity.

The differences between DAM and FVM solutions are best seen from the cross section plots (Figure 15). The agreement between the results is very good. The largest discrepancies are observed in the local extreme values of macrosegregation. The agreement is better as the inner and the outer radius of the hollow cylinder are increased. The matching is almost perfect for Case 3. 

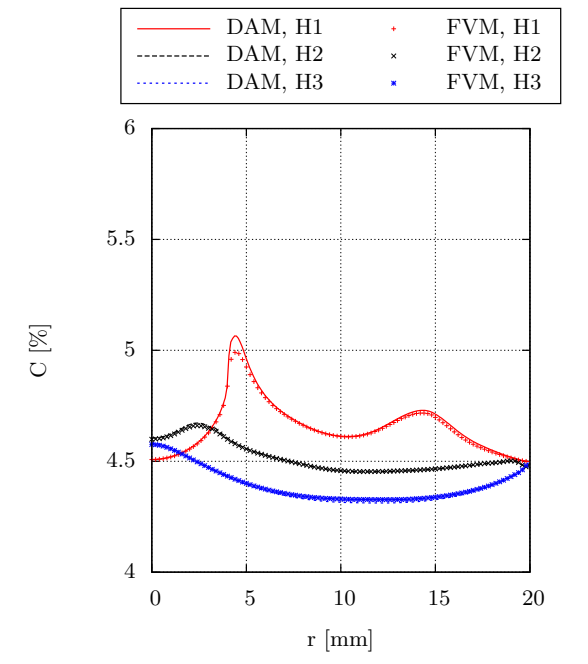

(a) Case 1, horizontal sections

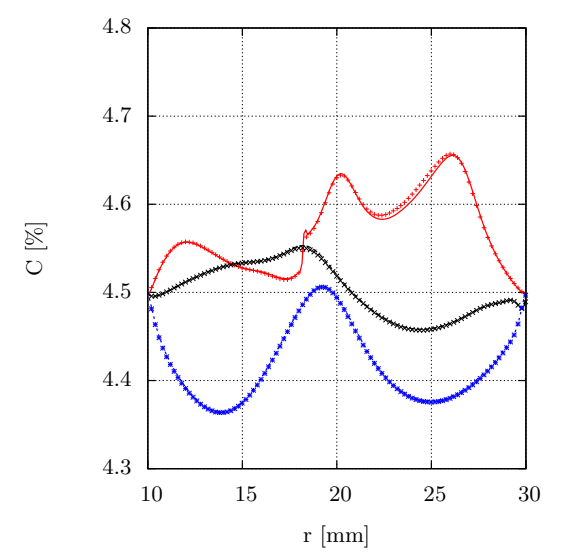

(c) Case 2, horizontal sections

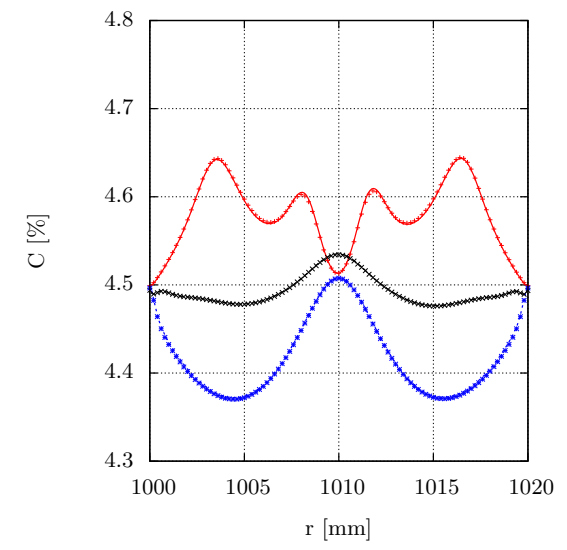

(e) Case 3, horizontal sections

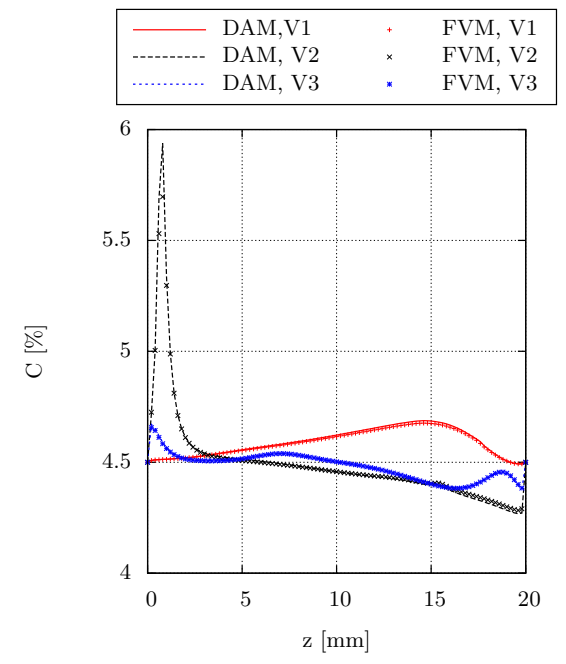

(b) Case 1, vertical sections

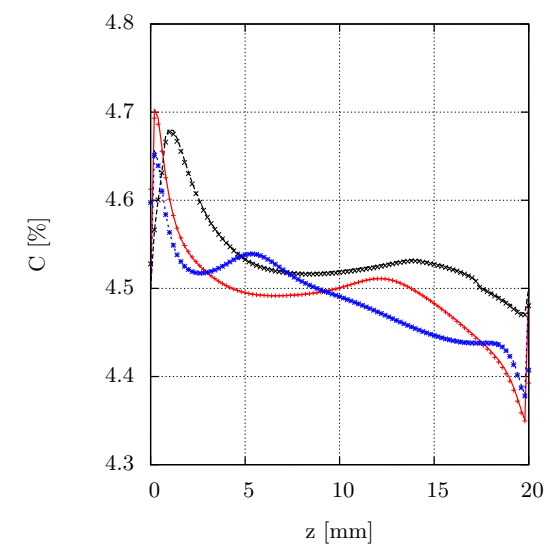

(d) Case 2, vertical sections

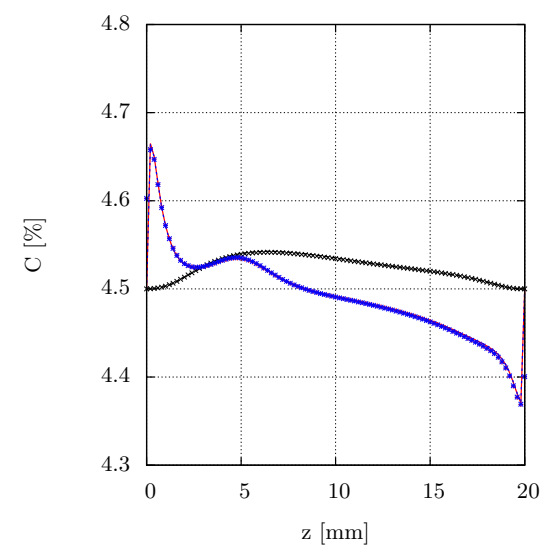

(f) Case 3, vertical sections

Figure 15: Cross sections of the final macrosegregation patterns for all three simulated cases. Horizontal and vertical sections are shown on the left and the right side, respectively. All results are for $240 \times 240$ computational node arrangement. Note that the range of the concentration axis is not the same for all cases. 
The tabulated results of the final macrosegregation pattern for DAM are given in Tables 2 and 3 for each horizontal and vertical cross section, respectively. The results are obtained with the finest density of computational nodes for all cases. Note that the results in the table are interpolated with the weighted least squares from the computational nodes to the reference nodes. Furthermore, the normalized root-mean-square deviance (NRMSD) between both results is calculated for the concentration values at the reference nodes

$$
\mathrm{NRMSD}=\frac{\mathrm{RMSD}}{C_{0}}=\sqrt{\frac{\sum_{i=1}^{n}\left(C_{i}^{\mathrm{DAM}}-C_{i}^{\mathrm{FVM}}\right)^{2}}{C_{0}^{2} n}},
$$

where $n$ is the total number of the reference points, listed in Tables 2 and 3. The superscripts DAM and FVM represent the solutions obtained with the diffuse approximate and the finite volume method. The NRMSD is equal to $0.181 \times 10^{-1}, 0.378 \times 10^{-3}$ and $0.272 \times 10^{-3}$ on the $240 \times 240$ node arrangement for Cases 1, 2 and 3, respectively. The NRMSD is small for all cases and confirms the good agreement between both methods. The agreement is better for higher values of inner radius, when the conditions are similar to the ones in a square cavity ( $x-y$ coordinate system). The largest differences between model predictions are for Case 1 in the area, where the concentration gradient is at its largest.

Table 2: Tabulated results of the diffuse approximate method for each horizontal cross section.

\begin{tabular}{|c||c|c|c||c|c|c||c|c|c|}
\hline \multicolumn{1}{|c||}{} & \multicolumn{3}{c||}{ Case 1 } & \multicolumn{3}{c||}{ Case 2 } & \multicolumn{3}{c|}{ Case 3} \\
\hline$r$ & $H_{1}$ & $H_{2}$ & $H_{3}$ & $H_{1}$ & $H_{2}$ & $H_{3}$ & $H_{1}$ & $H_{2}$ & $H_{3}$ \\
\hline 0.000 & 4.500 & 4.500 & 4.500 & 4.500 & 4.500 & 4.500 & 4.500 & 4.500 & 4.500 \\
\hline 0.002 & 4.557 & 4.665 & 4.517 & 4.557 & 4.512 & 4.393 & 4.573 & 4.486 & 4.405 \\
\hline 0.004 & 4.835 & 4.606 & 4.431 & 4.537 & 4.529 & 4.365 & 4.636 & 4.480 & 4.373 \\
\hline 0.006 & 4.800 & 4.528 & 4.369 & 4.524 & 4.536 & 4.402 & 4.572 & 4.482 & 4.386 \\
\hline 0.008 & 4.667 & 4.487 & 4.331 & 4.522 & 4.552 & 4.479 & 4.605 & 4.506 & 4.448 \\
\hline 0.010 & 4.614 & 4.457 & 4.315 & 4.633 & 4.519 & 4.494 & 4.514 & 4.535 & 4.508 \\
\hline 0.012 & 4.645 & 4.453 & 4.312 & 4.585 & 4.477 & 4.419 & 4.607 & 4.504 & 4.447 \\
\hline 0.014 & 4.727 & 4.460 & 4.318 & 4.605 & 4.459 & 4.381 & 4.571 & 4.480 & 4.386 \\
\hline 0.016 & 4.647 & 4.473 & 4.342 & 4.655 & 4.465 & 4.382 & 4.636 & 4.478 & 4.374 \\
\hline 0.018 & 4.549 & 4.492 & 4.392 & 4.562 & 4.485 & 4.414 & 4.573 & 4.486 & 4.405 \\
\hline 0.02 & 4.500 & 4.500 & 4.500 & 4.500 & 4.500 & 4.500 & 4.500 & 4.500 & 4.500 \\
\hline
\end{tabular}


Table 3: Tabulated results of the diffuse approximate method for each vertical cross section.

\begin{tabular}{|c||c|c|c||c|c|c||c|c|c|}
\hline \multicolumn{1}{|c||}{} & \multicolumn{3}{c||}{ Case 1} & \multicolumn{3}{c||}{ Case 2 } & \multicolumn{3}{c|}{ Case 3} \\
\hline$z$ & $V_{1}$ & $V_{2}$ & $V_{3}$ & $V_{1}$ & $V_{2}$ & $V_{3}$ & $V_{1}$ & $V_{2}$ & $V_{3}$ \\
\hline 0.000 & 4.500 & 4.500 & 4.500 & 4.500 & 4.500 & 4.500 & 4.500 & 4.500 & 4.500 \\
\hline 0.002 & 4.520 & 4.613 & 4.518 & 4.541 & 4.632 & 4.521 & 4.528 & 4.513 & 4.528 \\
\hline 0.004 & 4.543 & 4.523 & 4.509 & 4.503 & 4.552 & 4.528 & 4.531 & 4.535 & 4.532 \\
\hline 0.006 & 4.569 & 4.504 & 4.530 & 4.492 & 4.524 & 4.536 & 4.526 & 4.542 & 4.526 \\
\hline 0.008 & 4.594 & 4.482 & 4.531 & 4.493 & 4.517 & 4.508 & 4.503 & 4.541 & 4.503 \\
\hline 0.010 & 4.623 & 4.457 & 4.502 & 4.501 & 4.520 & 4.490 & 4.491 & 4.535 & 4.491 \\
\hline 0.012 & 4.654 & 4.436 & 4.472 & 4.511 & 4.526 & 4.473 & 4.482 & 4.529 & 4.482 \\
\hline 0.014 & 4.683 & 4.411 & 4.428 & 4.497 & 4.532 & 4.455 & 4.471 & 4.523 & 4.471 \\
\hline 0.016 & 4.667 & 4.374 & 4.388 & 4.467 & 4.522 & 4.442 & 4.456 & 4.517 & 4.455 \\
\hline 0.018 & 4.560 & 4.315 & 4.432 & 4.429 & 4.495 & 4.440 & 4.435 & 4.508 & 4.435 \\
\hline 0.02 & 4.500 & 4.500 & 4.500 & 4.500 & 4.500 & 4.500 & 4.500 & 4.500 & 4.500 \\
\hline
\end{tabular}




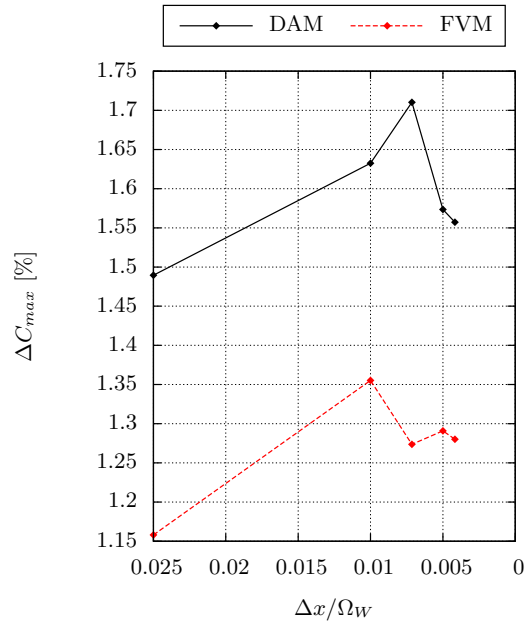

(a)

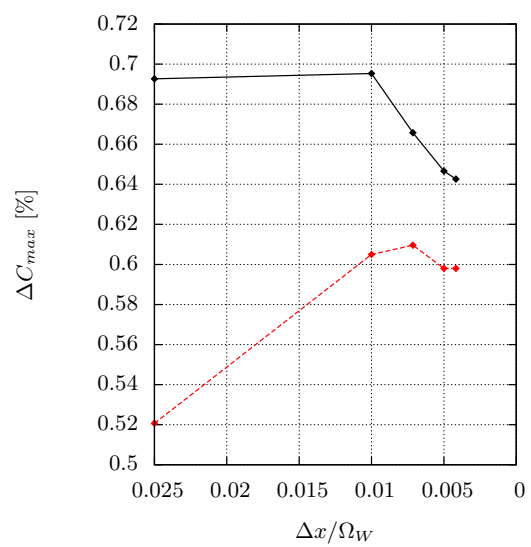

(c)

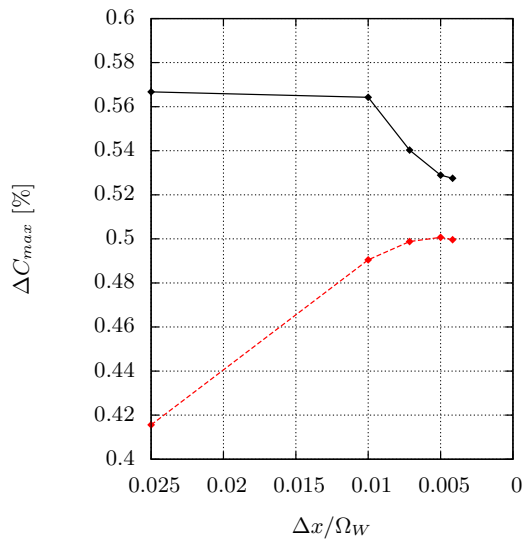

(e)



(b)

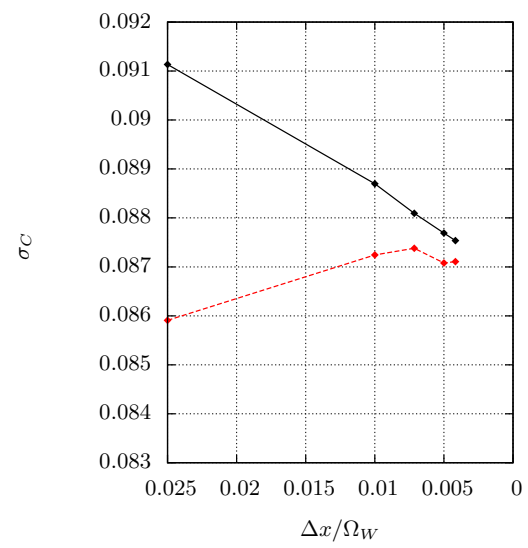

(d)

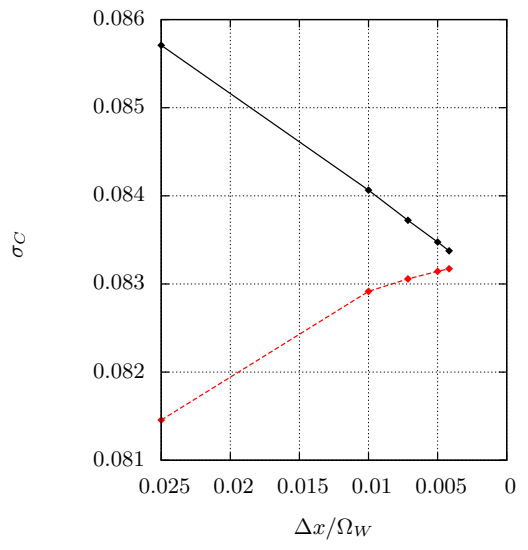

(f)

Figure 16: Node spacing convergence for varthables $\Delta C_{\max }$ (left) and $\sigma_{C}$ (right). Results are for Case $1(\mathrm{a}, \mathrm{b}), 2(\mathrm{c}, \mathrm{d})$ and $3(\mathrm{e}, \mathrm{f})$. 
The node spacing convergence of variables $\Delta C_{\max }$ and $\sigma_{C}$ is shown in Figure 16. We expect that the exact solution is obtained when the node spacing $\Delta x$ approaches zero. $\Delta C_{\max }$ is the maximum value of the segregation in the computational domain at the end of solidification $(t=50 \mathrm{~s})$

$$
\Delta C_{\max }=\max \left[C_{i}-C_{0}\right],
$$

where $i$ are the indices of the computational nodes in the domain for the $240 \times 240$ node arrangement. $\sigma_{C}$ is the global segregation index and represents the standard deviation of the concentration in the domain at the end of solidification.

$$
\sigma_{C}=\sqrt{\frac{1}{N \frac{r_{\text {in }}+r_{\text {out }}}{2}} \sum_{i=1}^{N} r_{i}\left(C_{i}-C_{0}\right)^{2}},
$$

where $C_{i}$ is the final segregation in node $i$ and $N$ is total number of nodes in the global domain used for computation. The node spacing convergence is evaluated with $\Delta C_{\max }$ and $\sigma_{C}$ for both numerical methods. Figure 16 shows that both numerical models converge for all three cases. The results confirm that the solution of the problem converges when the number of the computational nodes is increased. This is true for both numerical methods and for all cases. The differences in the calculated $\Delta C_{\max }$ values are slightly larger then in $\sigma_{C}$ values. This is because $\Delta C_{\max }$ lies in a very localised zone of positive segregation and is extremely sensitive to the computational node arrangement and discretisation method.

\section{Conclusions}

This is the first quantitative evaluation of the diffuse approximate method on a solidification test case solved in a cylindrical coordinate system. The simulations are performed for a solid and two hollow cylinder geometries with the use of an uniform computational node arrangement. Different number of the computational nodes are used to show the convergence of the results for the evaluated cases. The same solidification problem is solved with the well established finite volume method. The results of both methods are analysed and compared in this paper.

The results have shown a good agreement of simulations performed with both methods. This confirms the suitability of DAM for prediction of macrosegregation in a cylindrical coordinate systems. This is of particular 
interest for the industrial applications in axisymmetry. This macrosegregation test is evaluated in order to verify the use of DAM for the macrosegregation prediction in direct chill casting of aluminium billets. This can be done, because the solidification time and flow velocity magnitude in the test cases presented in this paper are of the same order of magnitude as in DC casting of billets [35].

\section{Acknowledgements}

This work was supported by the Slovenian Research Agency (BM and BŠ) (grant numbers J2-7384, P2-0162); Project MARTINA OP (grant number 20.00369); and Young Researchers programme (VH). A part of this

work was carried out as part of the GRADECET (GRAvity DEpendence of Columnar to Equiaxed Transition in TiAl Alloys) research project (MCF, $\mathrm{MZ}$ and $\mathrm{HC}$ ) and was funded by the European Space Agency (contract $4000114221 / 15 / \mathrm{NL} / \mathrm{PG}$ ). The view expressed herein can in no way be taken to reflect the official opinion of the European Space Agency.

\section{REFERENCES}

\section{References}

[1] A. Ludwig, M. Wu, A. Kharicha, On Macrosegregation, Metallurgical and Materials Transactions A 46 (11) (2015) 4854-4867. doi:10.1007/ s11661-015-2959-4.

[2] H. Li, S. S. Mulay, Meshless Methods and Their Numerical Properties, CRC Press, Boca Raton, FL, 2013.

[3] G. Liu, Mesh Free Methods: Moving Beyond the Finite Element Method, CRC Press, Boca Raton, FL, 2009.

[4] V. Hatič, B. Mavrič, N. Košnik, B. Šarler, Simulation of Direct Chill Casting Under the Influence of a Low-Frequency Electromagnetic Field, Applied Mathematical Modelling 54 (2018) 170-188. doi:10.1016/j . apm.2017.09.034.

[5] G. Kosec, M. Založnik, B. Šarler, H. Combeau, A meshless approach towards solution of macrosegregation phenomena, Computers, Materials, \& Continua 22 (2) (2011) 169-195. 
[6] H. Combeau, M. Bellet, Y. Fautrelle, D. Gobin, E. Arquis, O. Budenkova, B. Dussoubs, Y. Du Terrail, A. Kumar, B. Goyeau, S. Mosbah, T. Quatravaux, M. Rady, C. A. Gandin, M. Založnik, A Numerical Benchmark on the Prediction of Macrosegregation in Binary Alloys, Vol. 2, The Minerals, Metals and Materials Society, San Diego (CA, USA), 2011, pp. 755-762. doi:10.1002/9781118062142.ch91.

[7] H. Combeau, M. Bellet, Y. Fautrelle, D. Gobin, E. Arquis, O. Budenkova, B. Dussoubs, Y. D. Terrail, A. Kumar, C.-A. Gandin, B. Goyeau, S. Mosbah, T. Quatravaux, M. Rady, M. Založnik, Analysis of a numerical benchmark for columnar solidification of binary alloys, IOP Conference Series: Materials Science and Engineering 33 (1) (2012) 012086. doi:10.1088/1757-899X/33/1/012086.

[8] G. Kosec, B. Šarler, Simulation of macrosegregation with mesosegregates in binary metallic casts by a meshless method, Engineering Analysis with Boundary Elements 45 (2014) 36-44. doi:10.1016/j. enganabound.2014.01.016.

[9] J. Ni, C. Beckermann, A volume-averaged two-phase model for transport phenomena during solidification, Metallurgical Transactions B 22 (3) (1991) 349-361. doi : 10.1007/BF02651234.

[10] C. Y. Wang, C. Beckermann, Equiaxed dendritic solidification with convection: Part I. Multiscale/multiphase modeling, Metallurgical and Materials Transactions A 27 (9) (1996) 2754-2764. doi:10.1007/ BF02652369.

[11] A. V. Reddy, N. C. Beckermann, Modeling of macrosegregation due to thermosolutal convection and contraction-driven flow in direct chill continuous casting of an $\mathrm{Al}-\mathrm{Cu}$ round ingot, Metallurgical and Materials Transactions B 28 (3) (1997) 479-489. doi:10.1007/ s11663-997-0115-2.

[12] M. J. M. Krane, F. P. Incropera, D. R. Gaskell, Solidification of ternary metal alloys-I. Model development, International Journal of Heat and Mass Transfer 40 (16) (1997) 3827-3835. doi:10.1016/ S0017-9310(97)00041-0.

[13] C. J. Vreeman, M. J. M. Krane, F. P. Incropera, The effect of freefloating dendrites and convection on macrosegregation in direct chill 
cast aluminum alloys: Part I: model development, International Journal of Heat and Mass Transfer 43 (5) (2000) 677-686. doi:10.1016/ S0017-9310 (99) 00174-X.

[14] H. G. Weller, G. Tabor, H. Jasak, C. Fureby, A tensorial approach to computational continuum mechanics using object-oriented techniques, Computers in Physics 12 (6) (1998) 620-631.

[15] C. T. Crowe (Ed.), Multiphase flow handbook, Mechanical engineering series, CRC : Taylor \& Francis, Boca Raton, FL, 2006.

[16] M. Bellet, H. Combeau, Y. Fautrelle, D. Gobin, M. Rady, E. Arquis, O. Budenkova, B. Dussoubs, Y. Duterrail, A. Kumar, C. A. Gandin, B. Goyeau, S. Mosbah, M. Založnik, Call for contributions to a numerical benchmark problem for 2d columnar solidification of binary alloys, International Journal of Thermal Sciences 48 (11) (2009) 2013-2016. doi:10.1016/j.ijthermalsci.2009.07.024.

[17] B. Nayroles, G. Touzot, P. Villon, Generalizing the finite element method: Diffuse approximation and diffuse elements, Computational Mechanics 10 (5) (1992) 307-318. doi:10.1007/BF00364252.

[18] H. Sadat, C. Prax, Application of the diffuse approximation for solving fluid flow and heat transfer problems, International Journal of Heat and Mass Transfer 39 (1) (1996) 214-218. doi:10.1016/S0017-9310(96) 85018-6.

[19] C. Prax, H. Sadat, P. Salagnac, Diffuse approximation method for solving natural convection in porous media, Transport in Porous Media 22 (2) (1996) 215-223. doi:10.1007/BF01143516.

[20] O. Bertrand, B. Binet, H. Combeau, S. Couturier, Y. Delannoy, D. Gobin, M. Lacroix, P. Le Quéré, M. Médale, J. Mencinger, H. Sadat, G. Vieira, Melting driven by natural convection A comparison exercise: first results, International Journal of Thermal Sciences 38 (1) (1999) 5-26. doi:10.1016/S0035-3159(99)80013-0.

[21] C. Prax, H. Sadat, E. Dabboura, Evaluation of high order versions of the diffuse approximate meshless method, Applied Mathematics and Computation 186 (2) (2007) 1040-1053. doi:10.1016/j.amc.2006. 08.059. 
[22] B. Šarler, R. Vertnik, J. Perko, Application of diffuse approximate method in convective-diffusive solidification problems, Computers, Materials \& Continua 2 (1) (2005) 77-83.

[23] C. Wang, H. Sadat, C. Prax, A new meshless approach for three dimensional fluid flow and related heat transfer problems, Computers \& Fluids 69 (2012) 136-146. doi:10.1016/j.compfluid.2012.08.017.

[24] G. Kosec, A local numerical solution of a fluid-flow problem on an irregular domain, Advances in Engineering Software 120 (2018) 36-44. doi:10.1016/j.advengsoft.2016.05.010.

[25] M. Amaouche, F. N. Bouda, H. Sadat, The onset of thermal instability of a two-dimensional hydromagnetic stagnation point flow, International Journal of Heat and Mass Transfer 48 (21-22) (2005) 4435-4445. doi:10.1016/j.ijheatmasstransfer.2005.05.003.

[26] K. Reuther, M. Rettenmayr, Simulating phase transformations by a meshless method with front tracking, Acta Materialia 60 (5) (2012) 2128-2134, wOS:000302835300023. doi:10.1016/j.actamat. 2011. 12.026.

[27] N. Talat, B. Mavrič, V. Hatič, S. Bajt, B. Šarler, Phase field simulation of Rayleigh-Taylor instability with a meshless method, Engineering Analysis with Boundary Elements 87 (2018) 78-89. doi: 10.1016/j.enganabound.2017.11.015.

[28] N. Talat, B. Mavrič, G. Belšak, V. Hatić, S. Bajt, B. Šarler, Development of meshless phase field method for two-phase flow, International Journal of Multiphase Flow 108 (2018) 169-180. doi: 10.1016/j.ijmultiphaseflow.2018.06.003.

[29] V. Hatič, B. Mavrič, B. Šarler, Simulation of a macrosegregation benchmark with a meshless diffuse approximate method, International Journal of Numerical Methods for Heat \& Fluid Flow 28 (2) (2018) 361-380. doi:10.1108/HFF-04-2017-0143.

[30] B. Šarler, R. Vertnik, Meshfree explicit local radial basis function collocation method for diffusion problems, Computers \& Mathematics with Applications 51 (8) (2006) 1269-1282. doi:10.1016/j.camwa.2006. 04.013. 
[31] G. Kosec, B. Šarler, Solution of thermo-fluid problems by collocation with local pressure correction, International Journal of Numerical Methods for Heat \& Fluid Flow 18 (7-8) (2008) 868-882. doi: 10.1108/09615530810898999.

[32] K. Mramor, R. Vertnik, B. Šarler, Low and intermediate Re solution of lid driven cavity problem by local radial basis function collocation method, Computers, Materials, \& Continua 36 (1) (2013) 1-21.

[33] K. Mramor, R. Vertnik, B. Šarler, Simulation of natural convection under the influence of magnetic field by Explicit Local Radial Basis Function Collocation Method, Computer Modeling in Engineering and Sciences 92 (4) (2013) 327-352, oCLC: 821104077.

[34] J. Perko, B. Šarler, Weight function shape parameter optimization in meshless methods for non-uniform grids, Computer Modeling in Engineering \& Sciences 19 (1) (2007) 55-68.

[35] M. Založnik, S. Xin, B. Šarler, Verification of a numerical model of macrosegregation in direct chill casting, International Journal of $\mathrm{Nu}-$ merical Methods for Heat \& Fluid Flow 18 (3/4) (2008) 308-324. doi:10.1108/09615530810853600. 


\section{Appendix A. Definition of the operators}

Definitions of the operators used in equations of the physical model are presented in this appendix. Definitions are given for a general scalar field $f$ and a vector field $\mathbf{F}$ in cylindrical coordinate system for axial symmetry.

Gradient of a scalar

$$
\nabla f=\frac{\partial f}{\partial r} \mathbf{e}_{r}+\frac{\partial f}{\partial z} \mathbf{e}_{z}
$$

Divergence of a vector

$$
\nabla \cdot \mathbf{F}=\frac{1}{r} \frac{\partial\left(r F_{r}\right)}{\partial r}+\frac{\partial F_{z}}{\partial z} .
$$

Laplace operator of a scalar

$$
\nabla^{2} f=\nabla \cdot(\nabla f)=\frac{1}{r} \frac{\partial}{\partial r}\left(r \frac{\partial f}{\partial r}\right)+\frac{\partial^{2} f}{\partial z^{2}} .
$$

Laplace operator of a vector

$$
\nabla^{2} \mathbf{F}=\left(\nabla^{2} F_{r}-\frac{F_{r}}{r^{2}}\right) \mathbf{e}_{r}+\nabla^{2} F_{z} \mathbf{e}_{z} .
$$

